\title{
AVALIAÇÃO DA TERAPIA NUTRICIONAL EM UTI CIRÚRGICA NEONATAL DE UM HOSPITAL PEDIÁTRICO DE SALVADOR - BAHIA
}

\author{
EVALUATION OF NUTRITIONAL THERAPY IN NEONATAL SURGICAL ICU OF A \\ PEDIATRIC HOSPITAL IN SALVADOR - BAHIA
}

\author{
Rogério dos Santos e Santos ${ }^{1}$ \\ Aline Ramalho Lima ${ }^{2}$ \\ Luama Araújo dos Santos ${ }^{3}$
}

\begin{abstract}
RESUMO: Introdução: A terapia nutricional (TN) é um conjunto de procedimentos terapêuticos para manutenção ou recuperação do estado nutricional do paciente. A TN tem como objetivos prevenir e tratar a desnutrição, preparar o paciente para o procedimento cirúrgico, além de melhorar a resposta imunológica. Objetivo: Analisar a efetividade da terapia nutricional e o ganho ponderal dos recém-nascidos internados numa unidade de terapia intensiva (UTI) cirúrgica neonatal de um hospital pediátrico de Salvador-BA. Material e Métodos: Estudo longitudinal, quantitativo, observacional e prospectivo, com a utilização de dados dos prontuários de neonatos de ambos os sexos internados na UTI cirúrgica, de um hospital pediátrico da cidade de Salvador-BA, no período entre fevereiro a junho de 2018. Foram utilizados protocolos padrão do hospital e da literatura para definir a adequação da dieta e ganho de peso. Resultados: A amostra foi composta por 59 pacientes com média de idade de $35,75 \pm 37$ dias, sendo a maior parte do sexo masculino (72,9\%). A adequação entre o prescrito e infundido foi de $96,4 \%$ que atingiram pelo menos $80 \%$ do volume infundido, $89 \%$ dos pacientes atingiram 100\% do prescrito. Apenas 3,6\% atingiram um percentual menor que $80 \%$. Os pacientes em uso de leite materno obtiveram um ganho ponderal médio de $173 \mathrm{~g}$, representando o maior entre os pacientes estudados. Conclusão: Os neonatos participantes do estudo apresentaram um percentual adequado de infusão de dieta, sendo importante destacar a importância do leite materno para a recuperação e manutenção do estado nutricional.
\end{abstract}

Palavras-chave: Terapia nutricional. Evolução clínica. Estado nutricional. Dietoterapia. Leite materno.

\footnotetext{
${ }^{1}$ Nutricionista, graduado pela Faculdade São Salvador, residente em saúde da criança e do adolescente pela liga Álvaro Bahia contra a mortalidade infantil. E-mail: rogeriomirrorı@@gmail.com.

${ }^{2}$ Nutricionista pela Universidade Federal da Bahia, especialista em Nutrição Clínica pela Universidade Gama Filho, especialista em Nutrição materno infantil pelo IPGS, especialista em segurança do paciente pela FIOCRUZ.E-mail: alinerlima@hotmail.com.

${ }^{3}$ Nutricionista pela Universidade do Estado da Bahia (UNEB). Mestre em Processos Interativos dos Órgãos e Sistemas pela Universidade Federal da Bahia (PPgPIOS/UFBA), com ênfase em Imunologia e Genética, Doutoranda em Processos Interativos dos Órgãos e Sistemas pela Universidade Federal da Bahia (PPgPIOS/UFBA), com ênfase em Microbiota Intestinal e Especialista em Nutrição Clínica na Obesidade e Estética pela Universidade do Estado da Bahia (UNEB) e Especialista em Nutrição e Fitoterapia pela UNYLEYA. Membro da Sociedade Brasileira de Fisiologia (SBFis).E-mail: luamaaraujos@gmail.com.
} 
ABSTRACT: Introduction: Nutritional therapy (NT) is o set of therapeutic procedures for maintenance or recovery of the patient's nutritional status. TN aims to prevent and treat malnutrition, prepare the patient for the surgical procedure, and improve the immune response. Objective: To analyze the effectiveness of nutritional therapy and the weight gain of infants admitted to a neonatal surgical intensive care unit (ICU) at a pediatric hospital in Salvador, Bahia. Material and Methods: A longitudinal, quantitative, observational and prospective study using data from the charts of neonates of both sexes admitted to the surgical ICU of a pediatric hospital in the city of Salvador, Bahia, from February to June 2018 Standard hospital and literature protocols were used to define dietary adequacy and weight gain. Results: The sample consisted of 59 patients with mean age of $35.75 \pm 37$ days, most of them male $(72.9 \%)$. The adequacy between the prescribed and infused was $96.4 \%$ that reached at least $80 \%$ of the volume infused, $89 \%$ of the patients reached $100 \%$ of the prescribed. Only 3.6\% reached a percentage lower than $80 \%$. Patients in breast milk obtained an average weight gain of $173 \mathrm{~g}$, representing the highest among the patients studied. Conclusion: Neonates participating in the study presented an adequate percentage of diet infusion, and it is important to highlight the importance of breast milk for recovery and maintenance of nutritional status.

Keywords: Nutritional therapy. Clinical evolution. Nutritional status. Diet therapy. Breast milk.

\section{INTRODUÇÃO}

A terapia nutricional (TN) é um conjunto de procedimentos terapêuticos para manutenção ou recuperação do estado nutricional do paciente, sendo indicada nos casos em que o paciente tem o trato gastrointestinal inteiramente ou parcialmente funcionante, porém é impossibilitado a alimentação por via oral (SILVA et al., 2013). A TN tem como objetivos prevenir e tratar a desnutrição, preparar o paciente para o procedimento cirúrgico, melhorar a resposta imunológica, modular a resposta ao trauma clínico e cirúrgico, prevenir e tratar infecções e doenças não infecciosas, melhorar a qualidade de vida do paciente, reduzir o tempo de internação, reduzir a mortalidade e, consequentemente, reduzir custos hospitalares (MCCLAVE et al., 2013; DROVER et al., 2or1; WAITZBERG et al., 2006).

Domingues, Silva e Silveira (2016) referem que a TN no paciente crítico (em risco iminente de perder a vida ou função de órgão/sistema do corpo humano) visa fornecer os nutrientes necessários para atender às diferentes demandas fisiológicas, proteger os órgãos vitais, e amenizar a proteólise, com a intenção de diminuir o estresse fisiológico e promover a manutenção da imunidade.

Pacientes em terapia intensiva frequentemente apresentam inadequações no suporte nutricional, tanto pela sub ou superestimação das necessidades energéticas diárias, quanto pela introdução tardia da terapia nutricional enteral. Um estudo observacional prospectivo, desenvolvido por Santos et al. (2017) em duas UTIS, sendo uma clínica e outra cirúrgica, de um Hospital Público Terciário, foram verificadas inadequações entre a administração de calorias e proteínas, e a meta nutricional calculada sendo assim evidenciada a dificuldade em proporcionar uma real infusão 
próxima aos valores calculados. Gomes et al. (2017) realizaram um estudo de caráter descritivo e longitudinal, na UTI Cardiológica e UTI geral de um Hospital Universitário na cidade de São Luís,

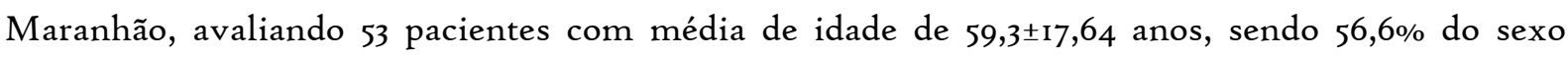
masculino e 34,4 do sexo feminino. A adequação entre o prescrito e o calculado foi (72,15\%) para caloria e 57,58\% para proteína, entre prescrito e infundido foi de 71,67\% tanto para caloria e proteína, a razão entre o calculado e infundido foi de $53,36 \%$ e 40,7\% de caloria e proteína. Foram observadas não conformidades em relação às metas pré-estabelecidas nos seguintes indicadores de qualidade: dias com oferta calórica administrada maior ou menor que 20\% da oferta prescrita em pacientes com nutrição enteral 25\%, dias com aporte proteico insuficiente no total de dias 49,37\%, e pacientes em jejum por mais de 24 horas $52,83 \%$. No entanto, foram encontradas inadequações na prescrição e oferta da nutrição enteral, principalmente proteica, bem como frequência elevada de jejuns inadequados e alta mortalidade.

O período neonatal, é uma fase considerada de vulnerabilidade à saúde dos neonatos por riscos biológicos, ambientais, sociais e culturais sendo necessário maior, atenção pelos profissionais de saúde para garantir o seu adequado desenvolvimento (PINHEIRO et al., 2or6). Contudo, frequentemente em UTI há necessidade de redução do volume infundido de alimentação, o que pode acarretar menor oferta calórico-proteica (FERNANDES et al., 2013).

Diante disso, analisar a administração da terapia nutricional e sua relação com o ganho de peso dos recém-nascidos permite demonstrar a importância de uma terapia nutricional efetiva para o adequado ganho de peso, influenciando no desenvolvimento e recuperação dos neonatos, para que assim possa garantir a recuperação do paciente, gerando menos custos por internamento hospitalar.

Esse trabalho teve como objetivo analisar a efetividade da terapia nutricional e a sua relação com o ganho de peso dos recém-nascidos internados numa unidade de terapia intensiva neonatal cirúrgica de um hospital pediátrico de Salvador-BA.

\section{Material e métodos}

Trata-se de um estudo, longitudinal, quantitativo, observacional e prospectivo, com a utilização de dados dos neonatos de ambos os sexos internados na Unidade de Terapia Intensiva (UTI), de um hospital pediátrico filantrópico da cidade de Salvador, BA, no período entre janeiro a junho de 2018.

Participaram da pesquisa neonatos, de ambos os sexos, com diferentes doenças, necessidades nutricionais e cirúrgicas admitidos na unidade em que foi realizada a coleta de dados, submetidos a $\mathrm{TN}$ enteral exclusiva via sonda por um período $>72$ horas, enteral concomitante, TN mista, leite 
materno exclusivo ou complementar via oral, ou por gavagem. Foi utilizado como critério de inclusão no estudo ter sido admitido na UTI neonatal no período da coleta dos dados (fevereiro a junho de 2018), sendo excluídos os pacientes com a ausência de dados importantes no prontuário, e como critério de não inclusão pacientes que foram a óbito antes de receberem dieta durante o período de internação.

A coleta de dados iniciou-se no dia de admissão do paciente na UTI, ou no dia em que houve a possibilidade de coleta de todos exigidos no estudo, sendo acompanhado até a alta da unidade ou óbito. Os dados foram coletados em balanços dos prontuários e tabulados em planilhas específicas do serviço de nutrição e programa STRATEC diariamente, nos quais se analisaram dados assistenciais, prescrição, e evoluções registrados por médicos, enfermeiras ou nutricionistas. Todos os dados coletados foram tabulados em planilhas de acompanhamento da TN do serviço de nutrição, em que se acompanhavam diariamente, sexo, idade, diagnóstico, desfecho clínico, comprimento, peso, infusão e prescrição de dieta, via de acesso, tipo de infusão da dieta, e eventuais intercorrências.

Todos os pacientes em uso de TN foram avaliados pela nutricionista para estimativa das necessidades energéticas e proteicas de acordo com a idade o diagnóstico e o protocolo da instituição. Não foram avaliadas necessidades nutricionais e o atingimento das mesmas nem Não percentuais de utilização das vias de administração no dia anterior á alta, pelo fato de que todos os pacientes recebiam alta com dieta via oral.

A adequação da dieta prescrita/ administrada foi obtida através de relação percentual (Prescrição/ Administração x Ioo) e avaliada, para identificar o atendimento da meta, e no desfecho final, sendo considerada adequada quando $\geq$ a $80 \%$, conforme proposto por Santana et al. (2016), sendo também meta diante dos registros internos. Foi avaliado também, o ganho ponderal desde o momento da admissão até o dia anterior à alta dos pacientes da unidade de internamento.

O banco de dados foi construído utilizando-se o programa Microsoft Office Excel versão 2010. Os dados foram tabulados em planilha no Microsoft Excel ${ }^{\circledR}$ e analisados com o auxílio do software para Windows Statistical Package for Social Science (SPSS) versão 21.o.

Este estudo foi aprovado pela Comissão Avaliadora de Ensino e Pesquisa (CAEP) do Hospital, e aprovado pelo comitê de ética em pesquisa da Universidade Federal da Bahia-UFBA, sob parecer de número 3.107.942.

\section{Resultados}

A amostra foi composta por 59 pacientes com média de idade de 35,75 \pm 37 dias, o tempo médio de permanência na UTI foi de $14,28 \pm 7$ dias, e a maior parte dessa população $(72,9 \%)$ era do sexo masculino. 
O diagnóstico mais frequente foi de cardiopatia $(28,8 \%)$, seguido de distúrbios do trato digestório $(25,4 \%)$, anomalias do sistema nervoso $(23,7 \%)$, distúrbios infecciosos $(8,5 \%)$, distúrbios em múltiplos sistemas (5,1\%), alteração do sistema respiratório (5,1\%), leucemia ( $1,7 \%)$ e hemangioma $(\mathrm{I}, 7 \%)$. As características da amostra foram descritas na Tabela $\mathrm{I}$.

\begin{tabular}{lll}
\hline Tabela I. Características da amostra do estudo & & \\
\hline Características & N & \\
Sexo & 43 & 72,9 \\
Masculino & $\mathrm{I} 6$ & $27, \mathrm{I}$ \\
Feminino & & \\
Doença de base & $\mathrm{I}$ & 28,8 \\
Cardiopatias & 5 & 8,5 \\
Distúrbios infecciosos & $\mathrm{I}$ & $\mathrm{I}, 7$ \\
Hemangiomas & $\mathrm{I}$ & $\mathrm{I}, 7$ \\
Leucemias & 3 & 5,1 \\
Distúrbios em múltiplos sistemas & $\mathrm{I}$ & 23,7 \\
Distúrbios em sistema nervoso central & 3 & 5,1 \\
Alterações em sistema respiratório & $\mathrm{I}$ & 25,4 \\
Distúrbios do trato digestório & & \\
\end{tabular}

As dietas administradas nos neonatos durante a admissão em sua grande maioria eram via sonda administradas por infusão gravitacional (50,8\%). Não foram avaliados percentuais de utilização das vias de administração no dia anterior á alta, pelo fato de que todos os pacientes recebiam alta com dieta via oral, o percentual da utilização das vias de administração está descrito na Tabela 2.

Tabela 2. Percentual de utilização das vias de administração de dietas

\begin{tabular}{|lcc|}
\hline Tipo de administração na admissão & N & $\%$ \\
\hline Dieta zero & II & 18,6 \\
\hline Oral & 9 & 15,3 \\
\hline Bomba de infusão & 30 & 50,8 \\
\hline Gravitacional & 9 & 15,3
\end{tabular}

Em relação ao peso ao nascer da população estudada, foi encontrado valor médio de $2,516 \pm$ 2,66o kg. O tipo de alimentação mais frequente na admissão foi fórmula infantil (69,5\%), seguido de dieta zero (18,6\%), aleitamento materno exclusivo (6,8\%) e aleitamento materno associado a fórmulas infantis $(5,1 \%)$. No dia anterior à alta, a dieta mais frequente continuou sendo a fórmula 
infantil (63,8\%), seguido de leite materno e fórmula infantil associadas $(22,4 \%)$, aleitamento materno exclusivo (ıo,3\%), dieta zero (I,7\%) e nutrição parenteral juntamente com fórmula infantil via enteral $(\mathrm{I}, 7 \%)$ como descrito na Figura I.

Figura r. Frequência dos tipos de dietas administradas nos neonatos presentes no estudo na admissão

A

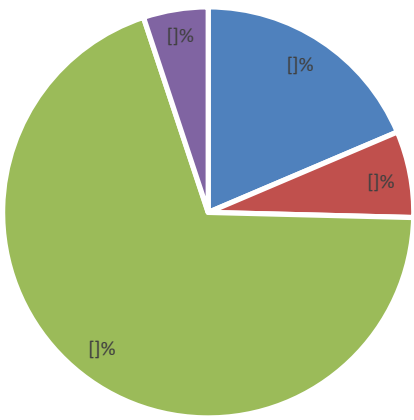

B

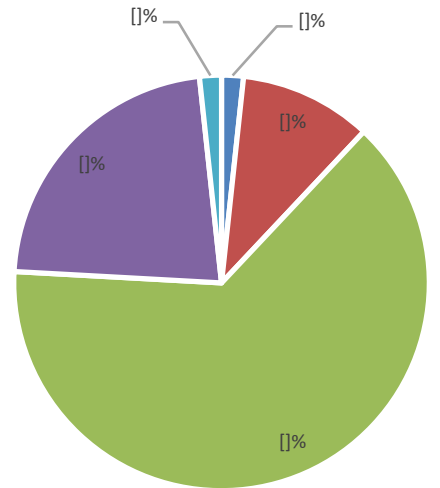

- dieta zero

- aleitamento

- fórmula

- leite + fórmula

- Parenteral

(A) e no dia anterior à alta (B)

As perdas de dietas não administradas na admissão resultaram em um valor médio de 29,05 $\pm 70,13 \mathrm{ml}$, e no dia anterior à alta, o valor médio de $15,14 \pm 66,60 \mathrm{ml}$. A adequação entre a dieta prescrita e infundida (pelo menos $80 \%$ da prescrição) na admissão foi de $73,2 \%$, enquanto na alta resultou em um percentual de $96,4 \%$. Ainda, no dia anterior à alta, 89,3\% dos indivíduos estavam com uma taxa de infusão de 100\% do volume prescrito pela equipe de terapia nutricional.

Ao final do internamento foi encontrada variação ponderal média na população do estudo de o, $146 \pm 0$,I04g. Os pacientes em uso exclusivo de leite materno sob livre demanda, tiveram um ganho ponderal médio de $173 \pm 0,390$ g, os que atingiram uma taxa de infusão adequada obtiveram um ganho ponderal médio de $149 \pm 0,533 g$, e os que não atingiram a adequação tiveram um ganho médio de II8 $\pm 0,216 \mathrm{~g}$ (Tabela 3).

Tabela 3. Ganho ponderal de acordo com adequação

\begin{tabular}{|lccc|}
\hline Adequação & N & Média & Desvio Padrão \\
\hline$>80 \%$ & 53 & 0,149 & 0,533 \\
\hline$<80 \%$ & 2 & 0,118 & 0,216 \\
\hline Aleitamento Materno & 4 & 0,173 & 0,390
\end{tabular}




\section{Discussão}

Na UTI neonatal foi observada que cerca de $79 \%$ dos neonatos eram do sexo masculino, e o diagnóstico mais frequente foi o de cardiopatia representando $28,8 \%$ dos casos. Segundo Catarino et al. (2017), as malformações congênitas representam a segunda principal causa de mortalidade em menores de um ano, sendo as cardiopatias congênitas as mais frequentes e com alta mortalidade no primeiro ano de vida.

Hoffman e Kaplan (2002) relatam que as cardiopatias congênitas podem afetar 4 a 50 por 1000 nascidos vivos, sendo que muitos desses casos necessitam de intervenção cirúrgica no primeiro ano de vida. Na ausência desta intervenção, pode haver agravamento do quadro clínico devido à sintomatologia, o que representa um fator chave para a internação de neonatos em UTI cirúrgica após o diagnóstico. A maioria das dietas foi ofertada aos neonatos via sonda administrada de modo gravitacional $(50,8 \%)$. Na prática clínica da UTI neonatal estudada, percebe-se que a indicação da via orogástrica é mais frequente e que a mesma é na maioria das vezes administrada por via gravitacional. Uma das principais vantagens da administração orogástrica é que a mesma melhor se aproxima da via fisiológica, mantendo o funcionamento e trofismo intestinal de maneira adequada (SOARES, 2018). Além disso, apresenta uma melhor tolerância, possibilitando uma rápida evolução para a via oral e do estado nutricional. Esta via ainda permite maior economia, uma vez que não são necessárias bombas de infusão e podem ser administradas pelas próprias mães, fortalecendo o vínculo mãe e filho. Ademais das referidas vantagens, a utilização da sonda orogástrica é preferível por preservar as vias aéreas superiores dos neonatos (NUNES et al., 2014).

Os valores de adequação de dieta mostraram que no dia da admissão os pacientes tiveram uma maior quantidade de dieta não infundida $(-29,05 \mathrm{ml})$ do que na alta $(-15,14 \mathrm{ml})$. Isso se justifica uma vez que a nutrição enteral deve sempre ser iniciada com um valor mínimo e este fornecimento deve ser aumentado gradualmente de acordo com a tolerabilidade do paciente (BRASIL,2016).

Notou-se no presente estudo que quase a totalidade dos pacientes receberam percentual adequado de dieta $(>80 \%)$, segundo meta interna estabelecida pela equipe do serviço de nutrição do hospital segundo as recomendações de Santana et al. (2016). Teixeira et al. (2017) referem a adequação da dieta prescrita e infundida como um fator importante para promover a saúde, a diminuição do estresse fisiológico e manter a imunidade. Por isso torna-se fundamental o controle para que a dieta prescrita pelo nutricionista seja administrada adequadamente pela equipe de enfermagem (CAPOROSSI et al.,2018). É fundamental ressaltar o grande número de indivíduos (86\%) que atingiram o volume total prescrito pela equipe de nutrição, o que favorece ainda mais a evolução do quadro clínico do paciente e reforça a qualidade do serviço prestado pelo hospital. 
O uso de fórmulas infantis foi mais frequente no grupo estudado que o aleitamento materno. É importante frisar que a ingestão de leite materno pelo paciente é de livre demanda e que não é rotina do hospital avaliar essa administração aos pacientes, além da falta de sala de ordenha e banco de leite o que dificulta a oferta do mesmo se não for por via oral representando um elevado risco de contaminação. Por isso, esta avaliação foi realizada separadamente das demais. As fórmulas infantis são criadas para atender às necessidades nutricionais dos neonatos na impossibilidade de utilização do leite materno, tentando ao máximo se assemelhar a este alimento (EVELEENS et al., 2018).

Em UTI, frequentemente os pacientes apresentam alterações gastrointestinais o que muitas vezes exige o uso de fórmulas especiais, e o alto índice de indivíduos com problemas cardíacos e respiratórios (que dificulta o processo de sucção do leite materno) torna plausível a alta utilização de fórmulas no grupo estudado (QUARESMA et al., 2015). Entretanto, torna-se necessário enfatizar que a prática do aleitamento materno deve ser sempre enfatizada, trabalhando as dificuldades de se amamentar um neonato e cada vez mais conscientizando as mães da sua importância (ALMEIDA; LUZ; UED, 2015). Esta importância, inclusive, pode ser notada ao comparar o ganho ponderal médio dos indivíduos alimentados com fórmula e com leite materno, o que confirma que o uso do leite materno é de extrema importância para manutenção e evolução do estado nutricional e clínico dos neonatos (ROUW; VON GARTZEN; WEIBENBORN, 2018). O hospital onde se realizou a pesquisa não tinha sala de ordenha para as mães nem banco de leite para os mesmos serem armazenados havendo uma dificuldade da sua utilização até mesmo em outras unidades pelo risco de contaminação.

É importante reforçar que apenas um paciente estava em dieta zero e que apenas um paciente estava em uso de nutrição parenteral associado a fórmula infantil, sendo impossível a comparação destes resultados com os demais grupos de estudo. Em relação ao paciente em dieta zero, o mesmo se apresentava criticamente enfermo e com anasarca o que dificulta ainda mais essa comparação, pois não há referência para desconto do edema na população estudada.

Os pacientes que atingiram uma taxa de infusão adequada obtiveram um ganho ponderal médio maior que os neonatos que tiveram inadequações na dieta, sendo justificada pelo fato de que a dieta quando não infundida adequadamente causa um déficit energético - proteico levando o neonato ao ganho de peso insuficiente (EVELEENS et al., 2018).

\section{Considerações finais}

Os resultados apresentados mostraram que a terapia nutricional na UTI neonatal, quando bem administrada, é efetiva na melhora clínica do paciente, e é de extrema importância ressaltar que 
deve ser priorizada a oferta do leite materno. No entanto, para que a TN obtenha o sucesso esperado, é preciso que haja uma adequada interação da equipe multiprofissional para que assim todas as condutas nutricionais aplicadas ao paciente sejam discutidas antes de serem adotadas. Também, na tentativa de minimizar os erros na terapia nutricional, sugere-se a criação de um protocolo para prescrição nutricional de acordo com a situação do paciente.

Torna-se necessário mais pesquisas sobre o tema para melhorias no serviço, monitorando continuamente a qualidade da terapia nutricional na unidade terapia intensiva neonatal.

\section{Limitações do estudo}

No presente estudo, foram encontradas algumas limitações: I) Não foi avaliado o posicionamento da sonda dos indivíduos estudados; II) Não foram avaliadas as pausas de dietas e quais motivos seriam mais frequentes; III) Não foi avaliada a quantidade de proteína e de calorias prescritas para os neonatos internados. Além disso, a planilha diária de infusão de dietas estava incompleta em algumas partes, dificultando a coleta adequada dos dados; IV) Não há sala de ordenha, nem banco de leite no hospital analisado; V) Não foram coletados dados aos fins de semana e feriados.

Referências

ALMEIDA, Jordana Moreira de; LUZ, Sylvana de Araújo Barros; UED, Fábio da Veiga. Apoio ao aleitamento materno pelos profissionais de saúde: revisão integrativa da literatura. Revista Paulista de Pediatria, [s.1.], v. 33, n. 3, p.355-362, set. 2015.

BRASIL. Ministério da Saúde. Secretaria de Atenção à Saúde. Departamento de Atenção Especializada e Temática. Manual de terapia nutricional na atenção especializada hospitalar no âmbito do Sistema Único de Saúde - SUS. Brasília: Ministério da Saúde, 2016.

BOCCOLINI, Cristiano Siqueira et al. Breastfeeding during the first hour of life and neonatal mortality. Jornal de Pediatria, [s.1.], v. 89, n. 2, p.131-136, mar. 2013.

CATARINO, Camilla Ferreira et al. Registros de cardiopatia congênita em crianças menores de um ano nos sistemas de informações sobre nascimento, internação e óbito do estado do Rio de Janeiro, 2006-2010. Epidemiol. Serv. Saúde, Brasília , v. 26, n. 3, p. 535-543, sept. 2017.

DOMINGUES, Laila Cristina do Couto et al.Terapia nutricional enteral em pacientes críticos: uma revisão de literatura [Trabalho de Conclusão de Curso - Pós-Graduação]. Goiânia: curso de Pósgraduação em Nutrição Clínica e Esportiva (CEEN), Pontifícia Universidade Católica de Goiás; 2016 
DROVER, John W. et al. Perioperative Use of Arginine-supplemented Diets: A Systematic Review of the Evidence. Journal Of The American College Of Surgeons, [s.1.], v. 212, n. 3, p.385-399, mar. 2011.

EVELEENS, R. D. et al. Weight improvement with the use of protein and energy enriched nutritional formula in infants with a prolonged PICU stay. Journal Of Human Nutrition And Dietetics, [s.1.], p.I-8, I4 out. 2018.

FERNANDES, Vanessa Pacini Inaba et al. Nutrição enteral em pediatria. Residência Pediátrica, Rio de Janeiro, v. 3, n. 3, p.67-75, set. 2013.

GOMES, Renata de Sousa et al. Qualidade da terapia nutricional enteral em unidades de terapia intensiva. Braspen journal: sociedade brasileira de nutrição parenteral e enteral. Maranhão, p. 165169. 03 fev. 2017

HOFFMAN, Julien I.e; KAPLAN, Samuel. The incidence of congenital heart disease. Journal Of The American College Of Cardiology, [s.1.], v. 39, n. I2, p.1890-190o, jun. 2002.

MCCLAVE, Stephen A. et al. Summary Points and Consensus Recommendations From the North American Surgical Nutrition Summit. Journal Of Parenteral And Enteral Nutrition, [s.1.], v. 37, n. 5, p.99-105, set. 2013. Wiley.

MELO, Maria do Carmo Barros et al. O aleitamento materno inserido na telessaúde: revisão integrativa. Latin American Journal Of Telehealth, [s.1.], v. 5, n. 2, p.099-I20, 4 set. 2018.

MENDONÇA, Mayara Ribeiro de; GUEDES, Gleyciane. Terapia nutricional enteral em uma Unidade de Terapia Intensiva: prescrição versus infusão. Braspen J. Salvador, 17 jul. 2017· p. 54-57

NUNES, J. DE A. et al. Preferência dos profissionais da unidade de terapia intensiva neonatal pelo uso da sonda nasogástrica ou orogástrica. Distúrbios da Comunicação. ISSN 2176-2724, v. 26, n. 2, p. 316-326, 2014.

OMS. Grupo de estudo de referência de crescimento multicêntrico da OMS. Padrões de crescimento infantil da OMS: Comprimento / altura / idade, peso / idade, peso / comprimento, peso / altura e índice de massa corporal / idade: Métodos e desenvolvimento. Genebra: Organização Mundial da Saúde, 2006.

PAPAGEORGHIOU, Aris $\mathrm{T}$ et al. International standards for fetal growth based on serial ultrasound measurements: the Fetal Growth Longitudinal Study of the INTERGROWTH-2ist Project. The lancet, [s.1.], v. 384, n. 9946, p.869-879, set. 2014.

PINHEIRO, Josilene Maria Ferreira et al. Atenção à criança no período neonatal: avaliação do pacto de redução da mortalidade neonatal no Rio Grande do Norte, Brasil. Ciência \& Saúde Coletiva, [s.1.], v. 21, n. I, p.243-252, jan. 2016.

QUARESMA, Liliana et al. Nutrição entérica do lactente com cardiopatia congénita. Acta Pediátrica Portuguesa, v. 46, n. 2, p. I19-125, 2015

ROUW, Elien; VON GARTZEN, Aleyd; WEIBENBORN, Anke. Bedeutung des Stillens für das Kind. Bundesgesundheitsblatt - Gesundheitsforschung - Gesundheitsschutz, [s.1.], v. 6I, n. 8, p.94595I, 25 jun. 2018. 
SANTANA, Mariana de Melo Alves et al. Inadequação calórica e proteica e fatores associados em pacientes graves. Revista de Nutrição, [s.1.], v. 29, n. 5, p.645-654, out. 2016

SANTOS, Polianna R. et al. Inadequação calórico-proteica e associação com Indicadores de Qualidade em Terapia Nutricional na assistência ao paciente crítico. O Mundo da Saúde, [s.1.], v. 4I, n. 4, p.66I-672, 3I dez. 2017. Centro Universitario Sao Camilo - Sao Paulo

SILVA, Fernanda Marchetto da et al. O impacto da introdução precoce de terapia nutricional enteral na redução da morbimortalidade na Terapia Intensiva Pediátrica: uma revisão sistemática. Revista da associação médica brasileira, [s.1.], v. 59, n. 6, p.563-570, nov. 2013.

SIMÕES, Silvana Aparecida Ribeiro et al. Dieta enteral prescrita versus dieta infundida Prescribed enteral diet versus infused diet. Revista de Pesquisa: Cuidado é Fundamental Online, [s.l.], v. 9, n. 3, p.688-695, II jul. 2017.

SOARES, Lorena Sousa. AVALIAÇÃO DA RETIRADA NÃO ELETIVA DOS TUBOS OROGÁSTRICOS EM RECÉM-NASCIDOS PREMATUROS. 2018. IoI f. Tese (Doutorado) Curso de Enfermagem, Departamento de Enfermagem, Universidade Federal do Piauí (ufpi). Teresina, 2018.

VENTURA, Claudiane Maria Urbano; ALVES, João Guilherme Bezerra; MENESES, Jucille do Amaral. Eventos adversos em Unidade de Terapia Intensiva Neonatal. Revista brasileira de enfermagem, [s.1.], v. 65, n. I, p.49-55, fev. 2012.

WAITZBERG, Dan L. et al. Postsurgical Infections are Reduced with Specialized Nutrition Support. World Journal Of Surgery, [s.1.], v. 30, n. 8, p.1592-1604, 16 jun. 2006. 